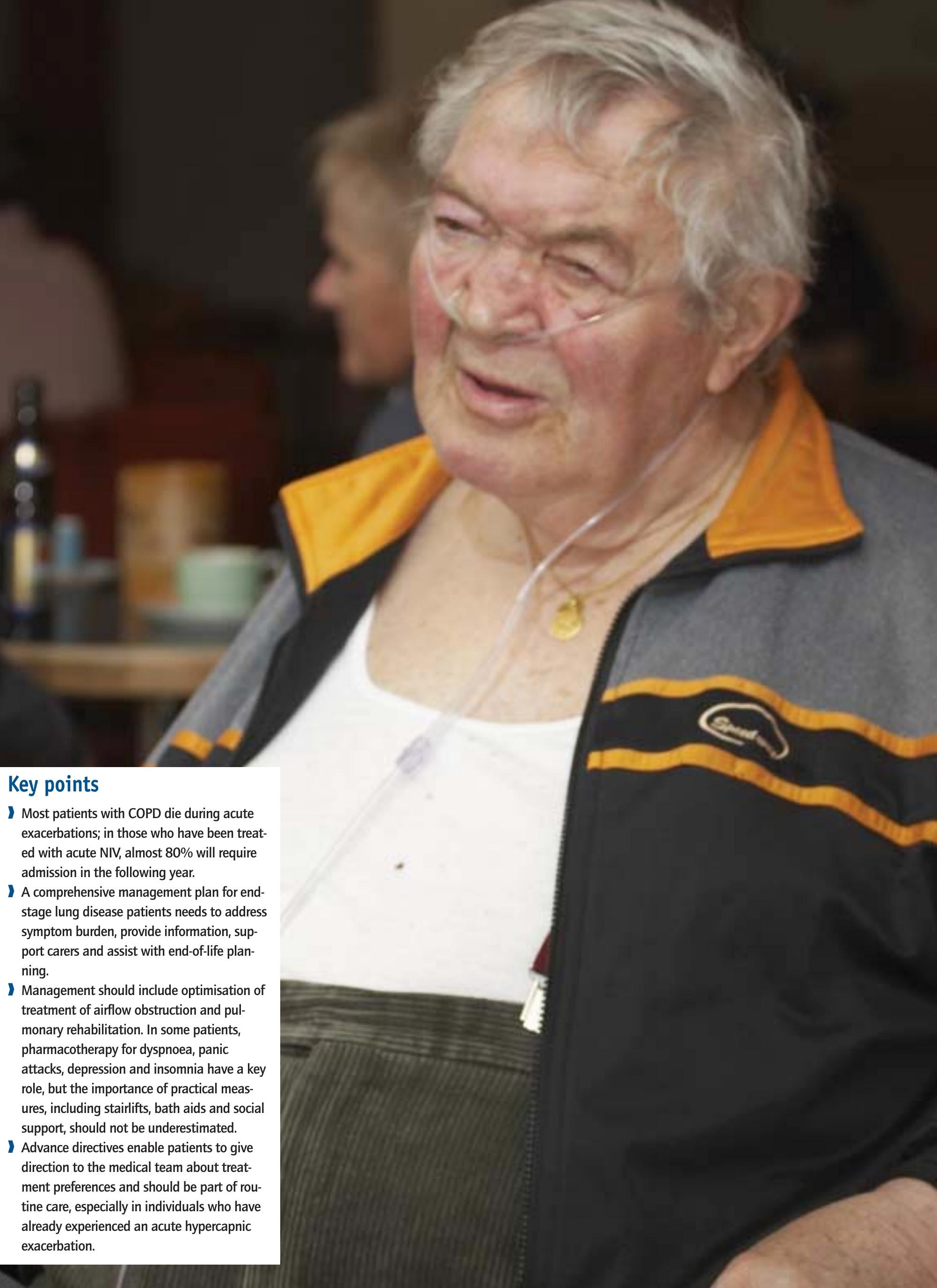




\section{Care of end-stage lung disease}

\section{Educational aims}

1 To raise awareness of the symptom load of end-stage lung disease.

1 To examine trajectories of end-stage disease.

I To discuss treatment strategies.

\section{Summary}

Individuals with end-stage lung disease have a substantial symptom burden, which is often poorly addressed compared to patients with malignant disease. Recognition of the disease trajectory in the last few years of life enables a symptom-based approach that combines palliative care with active treatment of exacerbations where appropriate. Discussion of advance directives is helpful for both the patient and the physician.

The management of end-stage lung disease has moved from the periphery to centre stage in respiratory medicine over the last few years, but approaches are often inconsistent. This is exemplified by the fact that current national, European and global chronic obstructive pulmonary disease (COPD) guidelines make few recommendations regarding end-of-life issues. Until recently, little research effort was directed towards the healthcare needs of COPD patients in their last years of life, despite the fact that as many patients with COPD die each year as those with lung cancer, and health decline occurs over a substantial period before that. Secondary (hospital) care has tended to focus on the treatment of airflow obstruction, acute exacerbations and, more recently, pulmonary rehabilitation, although provision of rehabilitation facilities does not meet requirements in many areas. Respiratory physicians are also often involved in the care of patients with respiratory insufficiency as a result of pulmonary fibrosis, or ventilatory pump failure due to muscular dystrophies, motor neurone disease or chest wall disorders. For all these groups, a unified approach linking community, primary and secondary care is often lacking.

\section{Disease trajectory of COPD in the last years of life}

It is important to realise that the clinical course in end-stage disease varies between conditions (figure 1). For COPD patients, a gradual deterioration in airflow obstruction and activity levels, punctuated by acute exacerbations, is the norm, which differs from the more predictable decline in lung cancer patients.

In a study of COPD deaths in London (UK), ELKINGTON et al. [1] found that 55\% of the survey group were male with an average age of 77 years. Demographic data were obtained from the person who reported the death. A remarkably high level of symptoms was reported in the 12 months before death:
The ERS designates this educational activity for a maximum of 1 CME credit. For information on how to earn CME credits, see page 380.

\section{A.K. Simonds \\ Correspondence: \\ Royal Brompton Hospital \\ Sydney Street \\ London SW3 6NP \\ UK \\ Fax: 442073518911 \\ E-mail: A.Simonds@rbht.nhs.uk}




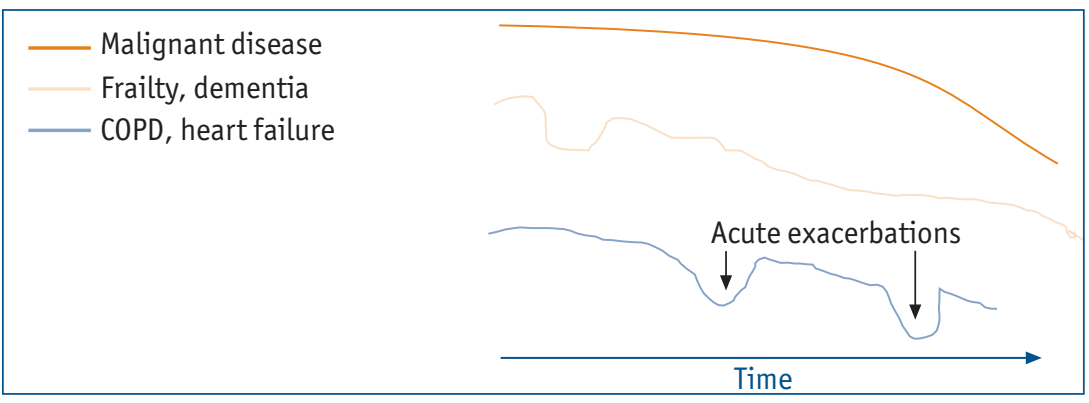

Figure 1

Predicting the natural history of end-stage conditions. The diagram shows the trajectories of some illnesses and patients' functional decline. interviewed by ELKINGTON et al. [1] were not aware that the deceased was likely to die. The carers felt that $\sim 63 \%$ of the deceased realised definitely or probably that they might die; however, a substantial number of patients were unclear about their own prognosis. Clearly, these individuals are unable to plan adequately for death if they do not know it is imminent. Regarding the place of death, it is also important to note that significantly more carers of subjects who died at home felt this was the right place to die as opposed to informants of those who died in hospital $(p=0.008)$. In England, a current Department of Health initiative is aiming to improve end-of-life care by ensuring more patients die in the place of their choice (usually home).

CHu et al. [2] have recently shown that the prognosis is poor in individuals who receive noninvasive ventilation (NIV) for acute hypercapnic exacerbations of COPD. In this study of 110 survivors of an acute episode of ventilatory failure treated with NIV (average age 73 years), virtually $80 \%$ required re-admission, mortality rate was $49 \%$ and $>63 \%$ had experienced another lifethreatening event in the following year. These patients spent an average of $12 \%$ of the following year in hospital. Symptom load (Medical Research Council (MRC) dyspnoea score) was predictive of short survival. These findings are consistent with another study that suggested hypercapnic COPD patients have a worse prognosis [3]. The results of these studies enable us to target patients in whom end-of-life strategies and discussions are entirely appropriate. Other opportunities to raise this subject are during pulmonary rehabilitation sessions, although general topics are easier to address in group meetings than specific management plans. For example, HEFFNer et al. [4] carried out a two-site prospective evaluation of advance directive education during a pulmonary rehabilitation course to assess the effects on completion of: 1) living wills; 2) durable powers of attorney (i.e. identifying a proxy decision maker); 3) patient-physician discussion about end-of-life issues; 4) decisions about life support; and 5) patient impression that their physician understood their end-of-life preferences. The group that received education on these topics was subsequently significantly more likely to discuss these issues, complete advance directives and feel more assured that their physicians understood their preferences. It has been demonstrated that COPD patients in particular require further information on their disease, its likely course and treatment from their doctors, but may not necessarily raise these 
topics without prompting. The way in which the topics are raised is also important. Most individuals welcome discussion, but this is usefully directed towards the symptom-control approach throughout the remainder of the patient's life, rather than an exclusive focus on their death. Striking a realistic and kindly balance between maintaining hope and a pragmatic expectation of deterioration is part of the clinical judgement healthcare workers should exercise and adapt to the individual.

\section{What patients want to know}

FRIED et al. [5] have examined treatment preferences regarding life-sustaining interventions in a range of patients with limited life expectancy due to cancer, congestive cardiac failure or COPD. Individuals aged $\sim 73$ years were asked whether they would wish to receive a given treatment if the outcome was certain or if there were differing likelihoods of an adverse outcome. Crucially, participants were able to balance the outcome against the burden of treatment (e.g. length of hospital stay, invasiveness of procedure). For example, a low-burden treatment that returned the individual to previous functioning level would be accepted by nearly all participants (98.7\%), but $11.2 \%$ would not accept this option if the treatment had a high burden. Conversely, if the likely outcome was survival but with severe functional or cognitive impairment, $74.4 \%$ and $88.8 \%$ would not want to receive low- and high-burden treatment, respectively. There was no difference between choices between the diagnostic groups, although there was a trend for COPD and cancer patients to refuse high-burden therapy. This study demonstrated that patients can compute varying outcomes, and that the impact on functional and cognitive outcomes plays a greater part in preferences than in survival itself. This should help inform discussion with patients. Clearly, they also need to know the probable consequences of non-intervention to make a valid decision.

\section{Communication and advance directives}

Advance directives formulated and recorded by a competent individual enable that person to give direction to healthcare providers about their choice of treatment in particular circumstances.
These can cover treatments that individuals would wish to receive if they developed respiratory failure, whether they would wish to be resuscitated and how these decisions would change if they were, for example, senile or unlikely to regain consciousness. Over the last $5-10$ years, growing numbers of people have taken up this option, although a UK survey has suggested that, so far, only $2 \%$ of the population has completed these.

Recent work has examined barriers to end-oflife discussions between the patient and the care team. In a focus group analysis of oxygendependent COPD patients and their physicians, KNAUF et al. [6] found that only $32 \%$ of patients had taken part in such discussions with their physician. Commonly cited barriers were "I'd rather concentrate on staying alive" and "I'm not sure which doctor will be taking care of me". Physicians ranked the most important barriers as "There was too little time during our appointment

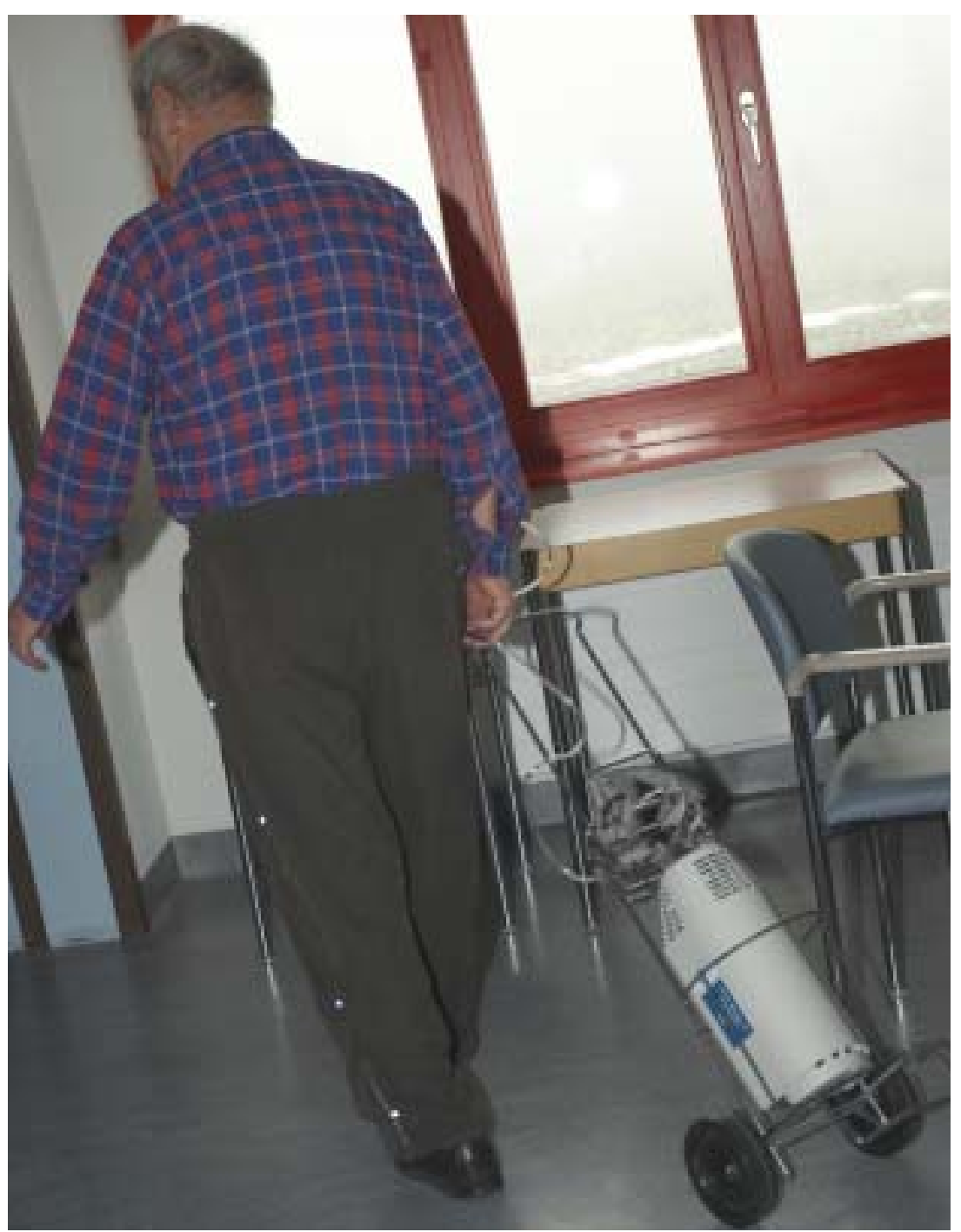




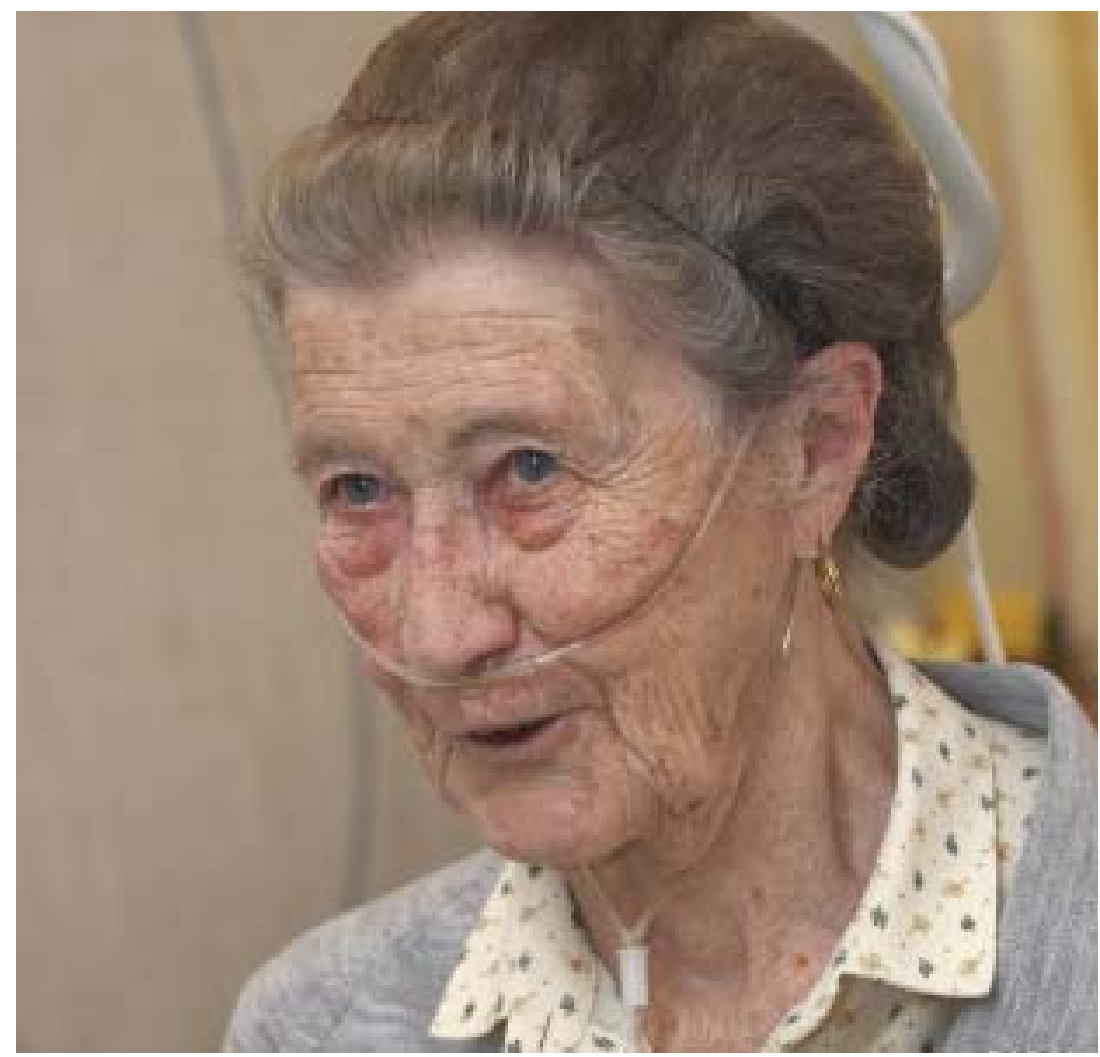

to discuss everything we should", or "I worry that discussing end-of-life care will take away his/her hope", and "The patient is not ready to talk about the care he/she wants if he/she is sick". It is evident that this topic is easier to defer on both sides. Conversely, the authors found a number of facilitators that made effective communication more likely. These included the patient's experience of friends or family who had died, the fact they trusted their physician, the feeling that their physician was good at caring for their lung disease and that he/she viewed them as person rather than focusing purely on their lung condition.

Although commonly viewed as a method of increasing autonomy and improving communication between the patient, family and healthcare team, there is some evidence that advance directives are not adhered to consistently. This may occur in circumstances that the healthcare team feel may not replicate those the patient had in mind when the advance directive was drafted [7]. Nonetheless, advance directives or living wills represent important steps forward in facilitating dialogue between the patient, family and the medical team.

Most advance directives concern decisions about treatment options that the individual would not wish to receive. There has been concern from some patient groups with neuromuscular disorders that if treatment is withdrawn due to a judgement about the quality of their life, this might not match their own perceived quality of life. It is clear that suitable advance directives stating which treatments they wish to receive may also be helpful. In the UK, a recent High Court judgement has confirmed that doctors need not provide futile care to patients.

\section{Symptom relief}

For relief of major symptoms, such as breathlessness, it goes without saying that airflow obstruction and hypoxaemia should be optimally treated according to evidence-based guidelines. Individuals should be carefully evaluated for common co-pathologies, such as left ventricular dysfunction, uncontrolled atrial fibrillation or anaemia, which are readily addressed. A recent meta-analysis [8] has assessed the role of opioids in the palliation of breathlessness in terminal illness. Of the 18 studies analysed, of which nine concerned nebulised opioid, JenNINGs et al. [8] found a small effect on reduction in breathlessness using non-nebulised opioids, but no overall beneficial effects on exercise tolerance or improvement in dypsnoea using nebulised delivery. Despite this, there are sporadic case reports on the successful use of nebulised opioid, e.g. nebulised fentanyl, for the palliation of dyspnoea in a cystic fibrosis patient with terminal lung disease in whom a reduction in air hunger and Borg score was noted [9]. An individual approach that carefully considers the bioavailability of the opioid and side-effects is clearly crucial. In some situations, side-effects such as sedation and constipation will outweigh benefit, and these considerations will also depend on whether treatment has been given in the last few days/hours of life or as a longer-term strategy.

Oxygen is commonly prescribed to reduce breathlessness, and should be used to correct hypoxaemia. There is little evidence that shortburst oxygen use is of benefit in COPD. STEVENSON and CALVERLEY [10] have shown that oxygen used on recovery from exercise in patients with severe COPD reduced the duration of dynamic hyperinflation, but, interestingly, this physiological gain did not decrease the perception of breathlessness or speed of recovery.

Pharmacological approaches are also valuable in treating depression, panic attacks and insomnia, all of which may exacerbate dypsnoea and/or reduce the quality of life. Serotonin 
selective re-uptake inhibitors may be particularly helpful in depression, panic and anxiety [11]. However, cognitive therapy and exploration of patient fears are important too. An overemphasis on drugs should be avoided. Discussion, reassurance and practical support, such as wheelchair, stair lifts and help for the main carer, should not be underestimated. Few hospice admissions are for end-stage lung disease, but hopefully this situation and the availability of respite care will improve [11].

\section{NIV as a palliative tool}

While much attention has been directed at the use of NIV to reduce mortality in acute exacerbations of COPD, early work has also shown that NIV reduces dyspnoea in this situation [12], and, therefore, NIV can be reasonably targeted towards symptom relief (figure 2). However, evidence of benefit is less established in steadystate circumstances. Here, careful attention should be focused on the aim of mechanical ventilation. This may be specifically to reduce dyspnoea, ameliorate symptoms related to hypercapnia, such as headaches and poor sleep quality, or potentially to buy time, e.g. for an individual to resolve family affairs or to allow time for a distant relative to attend the bedside. In all these situations, the goal of NIV should be decided in advance, so it should be quickly evident whether that aim has been achieved, or if the burden of NIV is outweighing any advantage. There have been few studies in this area, but Cuomo et al. [13] have shown that NIV significantly improved oxygenation and Borg dypnoea score in patients with end-stage solid cancer who develop acute respiratory failure. $\mathrm{pH}$ was only improved in hypercapnic patients. NIV can, therefore, be used reasonably to treat this group of patients in whom invasive ventilation is felt to be inappropriate [14].

There is increasing information on the use of NIV in end-stage neuromuscular conditions, such as motor neurone disease/amyotrophic lateral sclerosis. While uncontrolled studies consistently

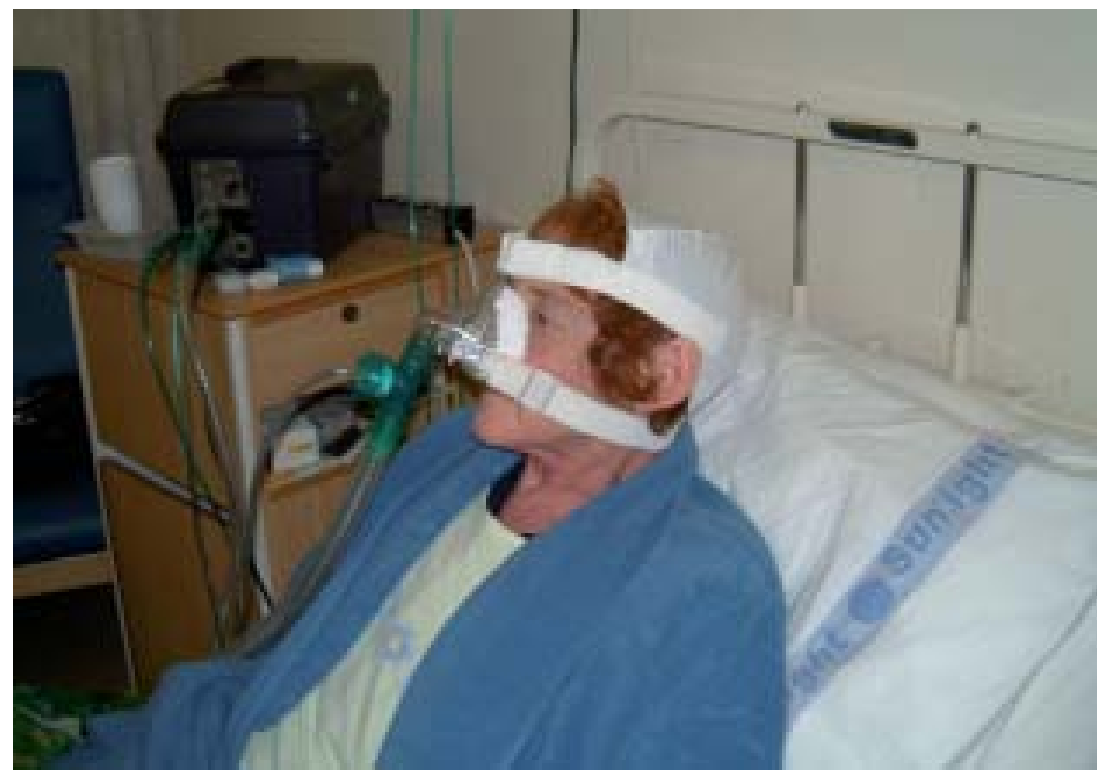

suggest NIV can increase survival and improve quality of life [15-17], the extent to which patients with bulbar-onset disease benefit is less clear, particularly as NIV may be more difficult to initiate and tolerate in this group [18]. However, a recent randomised controlled trail has confirmed that NIV improves survival and quality of life in motor neurone disease patients with mild or moderate bulbar disease, and although survival was not prolonged in those with severe bulbar involvement, a range of quality of life measures did improve [19].

\section{Conclusion}

All patients with chronic respiratory disease will ultimately enter a terminal phase unless they die of another cause. Care will improve with increasing acknowledgement by physicians and patients that this phase, although inevitable, is not untreatable. In general, patients value discussion of their treatment management and this needs to focus on the life that remains, as well as end-oflife planning. Simple direct questions such as "How do you see your chest problems developing in the future?" and "I'd like to know your views on how you would like to be treated if your condition deteriorated suddenly" should pave the way to a more open, rewarding and mutually beneficial patient-physician relationship.
Figure 2

A patient using NIV during an acute exacerbation of COPD.

\section{Educational questions}

Are the following statements true or false?

1. Short-burst oxygen therapy reduces dyspnoea in end-stage lung disease.

2. Following an acute exacerbation of COPD requiring NIV, $\sim 20 \%$ of patients are admitted to hospital in the following year.

3. An important physician barrier to the discussion of advance directives is a lack of time.

4. NIV is contraindicated as a palliative tool in COPD. 


\section{Suggested answers}

1. False

2. False

3. True

4. False

\section{References}

1. Elkington $H$, White $P$, Addington-Hall J, Higgs $R$, Edmonds $P$. The healthcare needs of chronic obstructive pulmonary disease patients in the last year of life. Palliat Med 2005; 19: 485-491.

2. Chu CM, Chan VL, Lin AWN, Wong WY, Leung WS, Lai CKW. Readmission rates and life threatening events in COPD survivors treated with non-invasive ventilation for acute hypercapnic respiratory failure. Thorax 2004; 59: $1020-1025$.

3. Connors AF, Dawson NV, Thomas C, et al. Outcomes following acute exacerbations of severe chronic obstructive lung disease. Am J Respir Crit Care Med 1996; 154: 959-967.

4. Heffner JE, Fahy B, Hilling L, Barbieri C. Outcomes of advance directive education of pulmonary rehabilitation patients. Am J Respir Crit Care Med 1997; 155: 1055-1059.

5. Fried TR, Bradley EH, Towle VR, Allore H. Understanding the treatment preferences of seriously ill patients. N Engl J Med 2002; 346: 1061-1066.

6. Knauft E, Nielsen EL, Engelberg RA, Patrick DL, Curtis JR. Barriers and facilitators to end-of life-care communication for patients with COPD. Chest 2005; 127: 2188-2196.

7. Thompson T, Barbour R, Schwartz L. Adherence to advance directives in critical care decision-making: vignette study. BMJ 2003; 327: 1011-1017.

8. Jennings AL, Davies AN, Higgins JPT, Broadley K. Opioids for the palliation of breathlessness in terminal illness. Cochrane Database Syst Rev 2001; 4: CD002066.

9. Graff GR, Stark JM, Grueber R. Nebulised fentanyl for palliation of dyspnoea in a cystic fibrosis patient. Respiration 2004; 71: $646-649$.

10. Stevenson $J C$, Calverley PMA. Effect of oxygen on recovery from maximal exercise in patients with obstructive pulmonary disease. Thorax 2004; 59: 668-672.

11. Hansen-Flaschen J. Chronic obstructive pulmonary disease: the last year of life. Respir Care 2004; 49: 90-97.

12. Bott J, Carroll MP, Conway JH, et al. Randomised controlled trial of nasal ventilation in acute ventilatory failure due to chronic obstructive airways disease. Lancet 1993; 341: 1555-1557.

13. Cuomo A, Delmastro M, Ceriana $P$, et al. Noninvasive mechanical ventilation as a palliative treatment of acute respiratory failure in patients with end-stage solid cancer. Palliat Med 2004; 18: 602-608.

14. Shee CD, Green M. Non-invasive ventilation and palliation: experience in a district general hospital and a review. Palliat Med 2003; 17: 21-26.

15. Lyall RA, Donaldson N, Fleming $T$, et al. A prospective study of quality of life in ALS patients treated with non-invasive ventilation. Neurology 2001; 57: 153-156.

16. Polkey MI, Lyall RA, Davidson AC, Leigh PN, Moxham J. Ethical and clinical issues in the use of home non-invasive ventilation for the palliation of breathlessness in motor neurone disease. Thorax 1999; 54: 367-371.

17. Bourke SC, Shaw PJ, Gibson GJ. Respiratory function vs sleep disordered as predictors of Q0L in ALS. Neurology 2001; 57: 2040-2044.

18. Aboussouan LS, Khan SU, Meeker DP, Stelmach K, Mitsumoto H. Effect of noninvasive positive-pressure ventilation on survival in amyotrophic lateral sclerosis. Ann Intern Med 1997; 127: 450-453.

19. Bourke SC, Tomlinson M, Williams TL, Bullock RE, Shaw PJ, Gibson GJ. Effects of non-invasive ventilation on survival and quality of life in patients with amyotrophic lateral sclerosis: a randomised controlled trial. Lancet Neurol 2006; 5: 140-147. 